\title{
Parental eating attitudes and indicators of healthy eating in a longitudinal randomized dietary intervention trial (the STRIP study)
}

\author{
Sanna Talvia 1,* , Leena Räsänen ${ }^{2}$, Hanna Lagström ${ }^{3}$, Susanna Anglè ${ }^{4}$, Maarit Hakanen ${ }^{1}$, \\ Minna Aromaa ${ }^{5}$, Lauri Sillanmäki ${ }^{1}$, Maiju Saarinen ${ }^{1}$ and Olli Simell ${ }^{6}$ \\ ${ }^{1}$ Research Centre for Applied and Preventive Cardiovascular Medicine, University of Turku, Kiinamyllynkatu 10, \\ FIN-20520 Turku, Finland: ${ }^{2}$ Division of Nutrition, Department of Food and Environmental Sciences, University of \\ Helsinki, Helsinki, Finland: ${ }^{3}$ Turku Institute for Child and Youth Research, University of Turku, Turku, Finland: \\ ${ }^{4}$ School of Public Health, University of Tampere, Tampere, Finland: ${ }^{5}$ City of Turku Health Care, Turku, Finland: \\ ${ }^{6}$ Department of Pediatrics, University of Turku, Turku, Finland
}

Submitted 15 June 2010: Accepted 22 March 2011: First published online 24 May 2011

\begin{abstract}
Objective: To examine the effects of child-oriented dietary intervention on parental eating attitudes and dietary behaviour.

Design: In the prospective, randomized Special Turku Coronary Risk Factor Intervention Project for Children (the STRIP study), a cohort of Finnish families took part in a nutritional intervention trial focused on the quality of their children's fat intake since the age of 8 months. Health-related and hedonic eating attitudes of the parents were measured after 10 years of dietary intervention using a validated Health and Taste Attitude Scales (HTAS) questionnaire ( $n$ 660). Parents' eating behaviour was studied using a $1 \mathrm{~d}$ food record ( $n$ 491).

Setting: Finland.

Subjects: Mothers and fathers ( $n$ 660) of the STRIP children.

Results: The parents of the intervention families had a higher level of interest in healthy eating compared with control parents. The interest in natural products or hedonic eating attitudes did not differ between the groups. The parents' general health interest was associated with low saturated fat intake, fruit and vegetable consumption, fibre intake and seeking pleasure in eating, but it was not associated with BMI. The intervention also improved the quality of dietary fat among parents with the lowest level of interest in healthy eating.

Conclusions: Parents' general health interest was associated with regular dietary counselling as well as with healthier food choice behaviour. However, the dietary intervention that focused especially on the quality of the child's fat intake also enhanced specific changes in the family's fat consumption without a high level of interest in healthy eating.
\end{abstract}

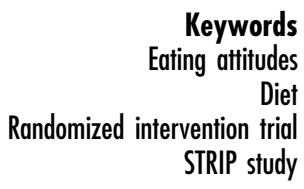

Keywords

Diet

STRIP study
To be effective, nutritional education aiming at prevention of diet-related diseases should begin in childhood $^{(1,2)}$. Recent evidence shows that eating behaviour and food choices are established early in life and show long-term stability ${ }^{(3)}$, and that childhood diet is a significant determinant of the cardiovascular quality of the adult $\operatorname{diet}^{(4)}$. Parents occupy a central position in the development of children's eating behaviour. Therefore, not only children but the whole family should be the focus of nutritional interventions ${ }^{(5,6)}$.

Finnish intervention families have been a part of the nutritional intervention trial of the prospective, randomized Special Turku Coronary Risk Factor Intervention Project for Children (the STRIP study) since their children were 8 months of age ${ }^{(7,8)}$. The main aim of the intervention was to influence the development of cardiovascular risk factors, especially fat intake, in childhood. It has been shown previously that the intervention influenced the food consumption and nutrient intake of both children and their parents ${ }^{(7-11)}$. Effects on serum lipids, lipoproteins and endothelial function have also been observed ${ }^{(9,10,12,13)}$. The intervention influenced the nutritional knowledge in parents, but the knowledge correlated poorly with dietary behaviour $^{(14)}$. Therefore, factors other than dietary knowledge, at least the rather theoretical knowledge measured, may better explain the differences in diet.

Many people acknowledge the importance of healthy eating $^{(15,16)}$; however, in practical situations pertaining to 
food choice, other factors such as convenience, price, mood or sensory appeal play a role as well. Appreciation of the sensory aspects of food seems to be at the centre of the development, maintenance and change of dietary patterns ${ }^{(17)}$. In a pan-European Union consumer attitudinal survey, one of the main barriers that consumers experienced when trying to eat healthier was giving up foods they liked ${ }^{(18)}$.

The aim of the present study was to examine eating attitudes in parents taking part in the STRIP study by using the Health and Taste Attitude Scales (HTAS). The HTAS is a validated self-assessment questionnaire measuring attitudes towards healthy and hedonic eating ${ }^{(19-21)}$. We analysed whether the regular dietary intervention during the first 11 years of the child's life and parents' BMI were associated with parents' attitudes towards eating. We also examined whether there was a correlation between interest in healthy eating and hedonic eating attitudes. Furthermore, we studied the association between interest in healthy eating and dietary indicators of healthy eating behaviour.

\section{Subjects and methods}

\section{Design and participants of the STRIP study}

The STRIP study is a prospective, randomized CHD risk factor intervention trial conducted in Finnish families. The families were recruited to the study by nurses at wellbaby clinics in the city of Turku, Finland, at the infants' routine 5-month visit between December 1989 and May 1992 as described ${ }^{(22)}$. The 1062 participating infants with their families were randomly allocated into an intervention group ( $n$ 540) or a control group $(n$ 522) at the 7 -month visit. The intervention families received regular, individualized dietary and lifestyle interventions aimed at reducing known CHD risk factors. They visited the study centre at 1-3-month intervals until the children were 2 years old and biannually thereafter. The control families, which did not receive any preventive intervention, were seen biannually until the children were 7 years old and once a year thereafter. After approximately $10 \cdot 5$ years of the intervention (when the child was 11 years old) 602 families visited the study centre: 280 and 322 from the intervention and control groups, respectively.

The percentage of families who withdrew from the study was similar between the intervention and control children and between boys and girls. The main reasons for loss-to-follow-up were changes in family situation or place of residence. The characteristics of children still participating in the STRIP study and those lost to followup have been compared repeatedly ${ }^{(12,13,23)}$ and no major differences have been found in weight, BMI, serum lipoproteins or dietary data.

At the beginning of the study an informed consent was obtained from the parents. No financial incentives were given to the families. The STRIP study was approved by the Ethics Committee of the Turku University and by the Turku University Hospital.

\section{Dietary intervention}

The purpose of the dietary intervention was to gradually increase parents' and later also children's knowledge of healthy eating and support the belief that children's dietary habits can be modified and that favourable changes in diet will improve health. Parents were encouraged to purchase and eat healthy food themselves and enlarge the repertoire of healthy foods offered to the children and their families. The main dietary aims were to reduce saturated fat intake and increase the unsaturated-to-saturated fat ratio (U:S). The optimal diet was defined to contain energy without any restrictions, fat $30 \%$ of energy (30-35\% of energy between 13 months and 2 years) and saturated fat $\leq 10 \%$ of energy with U:S of $2: 1$.

The biannual dietary intervention session with a nutritionist consisted of nutritional education with a specific dietary topic (e.g. saturated and unsaturated fat, visible and invisible fat, and role of fruit and vegetables and wholegrain products in healthy eating) and counselling dealing with the child's and family's diet. The total time of one intervention session for each family was approximately $30 \mathrm{~min}$. Depending on the family's circumstances, as well as their interests and time restrictions, one or both parents participated in the session. At the beginning of the study only parents participated actively in the intervention, but after the child turned 7 years old a separate educational session was organized for the child. The parents were carefully informed about the tasks the child had performed during the separate educational session.

Individualized dietary counselling was based mainly on the food consumption data of the child. Data were obtained using food records of four consecutive days close to each study visit. The family's general food choices such as type of milk and dietary fat were also discussed at each study visit. The family received both oral and written tailored feedback about the child's nutrient intake. Comparisons with the Finnish dietary recommendations were made, as well as suggestions on how to improve the quality of the child's diet.

Written material and brochures on the specific topic of each intervention session were given to the families during the session. After the age of 7 years, two letters with paper-pencil or cut-glue tasks and food preparation tasks were sent home between the two annual visits. Letters included a separate information sheet for parents in which the nutritional background information of the tasks was given in simple terms. The parents were also encouraged to discuss food choice and preparation issues with their child.

The control families did not routinely receive any detailed intervention focused on risk factors of atherosclerosis. The control families filled in a $4 \mathrm{~d}$ food record of the child before each study visit biannually until the child 
reached 7 years of age and once annually thereafter. Families received a short general feedback letter about the child's dietary intake, focusing on issues other than dietary fat (e.g. energy and sucrose intakes), to motivate them to maintain the food diary and take part in the study.

\section{Attitude measurements}

The eating attitudes of the parents were measured using the HTAS, an attitude measurement tool developed in Finland $^{(19)}$. The HTAS has been previously validated in Finnish, Dutch and British adults ${ }^{(20,21)}$. The original questionnaire consisted of twenty health-related and eighteen taste-related statements, which were scored on a 7-point Likert scale with the categories ranging from 'disagree strongly' (1) to 'agree strongly' (7). The original scales consisted of six subscales: 'General health interest', 'Light product interest', 'Natural product interest', 'Craving for sweet foods', 'Using food as a reward' and 'Pleasure'. Each subscale was composed of an equal number of positively and negatively worded statements in order to minimize the respondents' tendency to agree with the items.

Separate questionnaires were sent for the mothers ( $n$ 602) and fathers ( $n$ 599) of those families who visited the study centre when the child was 11 years old. Two questionnaires were also sent to families in which the parents had divorced/separated during the study years and hence did not live together. However, only those parents who had taken part in the study from the beginning were included in the analysis (i.e. new step-parents were excluded). Instructions on how to fill in the questionnaire, as well as a pre-paid envelope, were sent along with the questionnaires. Valid questionnaires were received from 369 mothers $(61 \cdot 3 \%$ of the questionnaires sent) and 241 fathers $(48.7 \%$ of the questionnaires sent). The mean age of the mothers and fathers was $40 \cdot 7$ (SD $4 \cdot 87$ ) years and $42 \cdot 4(\mathrm{SD} 4 \cdot 87)$ years, respectively.

\section{Measurement of parents' diet and BMI}

Parents' food consumption data were obtained using food records of $1 \mathrm{~d}$ close to the child's eleventh birthday, before the annual visit to the study centre. Diary forms were sent to the families 3-4 weeks before the follow-up visit. The parents were given written instructions on how to record food consumption, with exact descriptions of the amounts of all foods and drinks. They included instructions and drawings, which helped them to maintain records and estimate the amount of food. The amount was estimated mainly using household measures (spoons, cups, glasses). The parents also recorded the type, brand and preparation method of all foods consumed. The number of parents with a completed attitude questionnaire and a food record was 135 among the intervention mothers, ninety-three among the intervention fathers, 151 among control mothers and 112 among control fathers. Food consumption was calculated using a program (Micro Nutrica ${ }^{\circledR}$ ) developed at the Research
Center of the Social Insurance Institution, Turku, Finland. The program used in the STRIP study was updated with new recipes and foods that had come into the market and which were used by the families during the study.

Parents' weight and height were measured during the study visit. BMI was calculated as weight in kilograms divided by the square of height in metres $\left(\mathrm{kg} / \mathrm{m}^{2}\right)$. On the basis of BMI scores parents were grouped into normal weight $\left(18 \cdot 5-24 \cdot 9 \mathrm{~kg} / \mathrm{m}^{2}\right)$, overweight $\left(25 \cdot 0-29 \cdot 9 \mathrm{~kg} / \mathrm{m}^{2}\right)$ or obese $\left(\geq 30 \cdot 0 \mathrm{~kg} / \mathrm{m}^{2}\right)$, according to the recommendations of $\mathrm{WHO}^{(24)}$. One father and two mothers were excluded from the analysis because they were underweight (BMI $<18 \cdot 5 \mathrm{~kg} / \mathrm{m}^{2}$ ). Of the mothers, $10 \cdot 8 \%$ were obese ( $n 33$ ), $27 \cdot 1 \%$ were overweight ( $n$ 83) and $62 \cdot 1 \%$ were of normal weight ( $n$ 190). Of the fathers, $12 \cdot 7 \%$ were obese ( $n$ 25), 49.2\% were overweight $(n$ 97) and $38 \cdot 1 \%$ were of normal weight ( $n 75)$. No difference was observed in the prevalence of normal weight, overweight or obesity between the intervention and control parents.

\section{Statistical analysis}

The answers to the negative attitude statements of the HTAS were reversed so that the final high values indicated a positive attitude towards the phenomenon studied. Thereafter, health and hedonic items were analysed separately using the Maximum Likelihood factor analysis with Promax rotation. Items not clearly loading on a single factor and items with factor loadings $<0.4$ were excluded from further analysis. Factors with eigenvalues (i.e. the amount of variance explained by each factor) $>1$ were chosen for the factor selection process. The internal consistency of each factor was analysed with Cronbach's $\alpha$ statistics. The Spearman correlation was used to measure the associations between different subscales. Scores for factors 'General health interest', 'Light product interest' and 'Pleasure' were squared for linear model analyses because of negatively skewed distributions.

The association of intervention and gender on the mean scores of each subscale was assessed using ANOVA. The association between BMI and the attitude factor scores was analysed using regression and ANOVA models. BMI was modelled both as $\mathrm{kg} / \mathrm{m}^{2}$ and as weight groups using cutoff points described in the 'Subjects and methods' section. For further analyses of the association between 'General health interest' and dietary indicators of healthy eating, the respondents were first divided into three groups (low, medium and high) depending on their mean subscale values of 'General health interest' using the 33rd and 66th percentile points as cut-off values. These analyses were conducted using ANOVA. All ANOVA and regression analyses were performed with full models including gender and intervention group as covariates. Non-significant terms were excluded from the models using backward selection, with exclusion criteria of $P>0 \cdot 1$ for main effects and $P>0.05$ for interactions. The Tukey-Kramer adjustment was used in the post hoc analyses. Results are presented as 
Table 1 Factor structures, Cronbach's $\alpha$ and loadings and communalities of the attitude statements towards healthiness of foods in parents taking part in the STRIP study

\begin{tabular}{|c|c|c|c|c|}
\hline \multirow[b]{2}{*}{ Statements } & \multicolumn{3}{|c|}{ Loadings } & \multirow[b]{2}{*}{ Communality } \\
\hline & Factor 1 & Factor 2 & Factor 3 & \\
\hline \multicolumn{5}{|l|}{ General health interest } \\
\hline 1 I am very particular about the healthiness of food & 0.64 & -0.04 & $0 \cdot 18$ & 0.50 \\
\hline 2 I always follow a healthy and balanced diet & 0.58 & -0.05 & 0.08 & 0.36 \\
\hline 3 It is important for me that my diet is low in fat & 0.71 & $0 \cdot 10$ & 0.02 & 0.58 \\
\hline 4 It is important for me that my daily diet contains a lot of vitamins and minerals & $0 \cdot 61$ & -0.03 & $0 \cdot 13$ & 0.43 \\
\hline 5R I eat what I like and I do not worry about the healthiness of food & $0 \cdot 80$ & 0.01 & -0.09 & 0.60 \\
\hline 6R The healthiness of food has little impact on my food choices & 0.79 & 0.02 & -0.04 & 0.61 \\
\hline 7R The healthiness of snacks makes no difference to me & 0.67 & 0.00 & 0.07 & 0.49 \\
\hline $\begin{array}{l}\text { 8R I do not avoid any foods, even if they increase my cholesterol level } \\
\text { Cronbach's } \alpha=0 \cdot 88\end{array}$ & $0 \cdot 64$ & $0 \cdot 07$ & $-0 \cdot 11$ & $0 \cdot 41$ \\
\hline Variance explained $=10 \cdot 4 \%$ & & & & \\
\hline \multicolumn{5}{|l|}{ Light product interest } \\
\hline 1 I believe that eating light products keeps one's cholesterol level under control & 0.01 & 0.73 & 0.08 & 0.56 \\
\hline 2 I believe that eating light products keeps one's body in good shape & 0.00 & 0.59 & 0.07 & 0.36 \\
\hline $\begin{array}{l}3 \text { In my opinion, by eating light products one can eat more without getting too } \\
\text { many calories }\end{array}$ & $-0 \cdot 21$ & 0.47 & 0.07 & $0 \cdot 19$ \\
\hline 4R In my opinion, the use of light products does not improve one's health & 0.08 & 0.77 & -0.04 & 0.64 \\
\hline 5R In my opinion, light products do not help to reduce cholesterol levels & 0.06 & $0 \cdot 70$ & 0.03 & 0.53 \\
\hline $\begin{array}{l}\text { 6R I do not think that light products are healthier than conventional products } \\
\text { Cronbach's } \alpha=0 \cdot 81\end{array}$ & $0 \cdot 19$ & $0 \cdot 64$ & $-0 \cdot 14$ & 0.52 \\
\hline Variance explained $=7 \cdot 4 \%$ & & & & \\
\hline \multicolumn{5}{|l|}{ Natural product interest } \\
\hline 1 I do not eat processed foods because I do not know what they contain & 0.23 & $-0 \cdot 12$ & 0.42 & $0 \cdot 28$ \\
\hline 2 I try to eat foods that do not contain additives & 0.34 & $-0 \cdot 12$ & 0.53 & 0.49 \\
\hline 3 I would like to eat only organically grown vegetables & -0.04 & 0.07 & $0 \cdot 78$ & 0.59 \\
\hline 4R In my opinion, artificially flavoured foods are not harmful for my health* & 0 & $-0 \cdot 14$ & 0.38 & 0.16 \\
\hline $\begin{array}{l}\text { 5R In my opinion, organically grown foods are no better for my health } \\
\text { compared with those grown conventionally }\end{array}$ & -0.09 & $0 \cdot 22$ & 0.61 & 0.39 \\
\hline $\begin{array}{l}6 \mathrm{R} \text { I do not care about additives in my daily diett } \\
\text { Cronbach's } \alpha=0.72 \\
\text { Variance explained }=4.6 \%\end{array}$ & 0.43 & $-0 \cdot 10$ & 0.45 & 0.52 \\
\hline
\end{tabular}

STRIP, Special Turku Coronary Risk Factor Intervention Project for Children.

Reversed statements are marked with an ' $R$ ' after the statement number.

${ }^{*}$ This statement was removed after the second analysis because of the low factor loading $(<0 \cdot 40)$. The loadings and the communality shown here are from the second analysis.

†This statement was removed after the first analysis because it loaded to both Factors 1 and 3 . The loadings and the communality shown here are from the first analysis.

differences between least square means attained from the ANOVA models. Statistical analyses were conducted using the SAS statistical software package version $9 \cdot 2$ (SAS Institute, Cary, NC, USA).

\section{Results}

\section{Attitude questionnaire}

The item evaluation using factor loadings resulted in the retention of eighteen health-related items loading on three factors (Table 1) and sixteen hedonic items loading on four factors (Table 2). Cronbach's $\alpha$ ranged between 0.67 and 0.89 and the variance explained by each factor between $2 \cdot 7 \%$ and $15 \cdot 1 \%$ (Tables 1 and 2). There was a clear correlation ( $r=0.54$ ) between 'Understanding other people's craving' and 'Personal craving for sweet food'. 'General health interest' correlated moderately with 'Natural product interest' $(r=0 \cdot 44)$, 'Light product interest' $(r=0.33)$ and 'Pleasure' $(r=0 \cdot 29)$. 'Using food as a reward' correlated moderately with 'Personal craving' $(r=0 \cdot 42)$ and 'Understanding other people's craving' $(r=0 \cdot 35)$. All other correlations between the factors were $<0 \cdot 14$.

\section{Associations of eating attitudes with intervention and gender}

The intervention parents were more interested in healthy eating compared with control parents. In the 'General health interest' subscale, the intervention mothers rated higher than control mothers and the intervention fathers rated higher than control fathers (Table 3). In 'Light product interest' intervention fathers rated higher than control fathers but there was no difference between the mothers of the intervention and control groups. Further, there were no differences between intervention and control parents either in 'Natural product interest' or in any of the hedonic subscales.

An apparent gender effect was seen in both healthrelated and hedonic subscales. Mothers scored higher than fathers in 'General health interest', in 'Natural product interest' and in all hedonic subscales (Table 3). However, in 'Light product interest', only control mothers rated higher than control fathers. 
Table 2 Factor structures, Cronbach's $\alpha$ and loadings and communalities of the attitude statements towards hedonic aspects of foods in parents taking part in the STRIP study

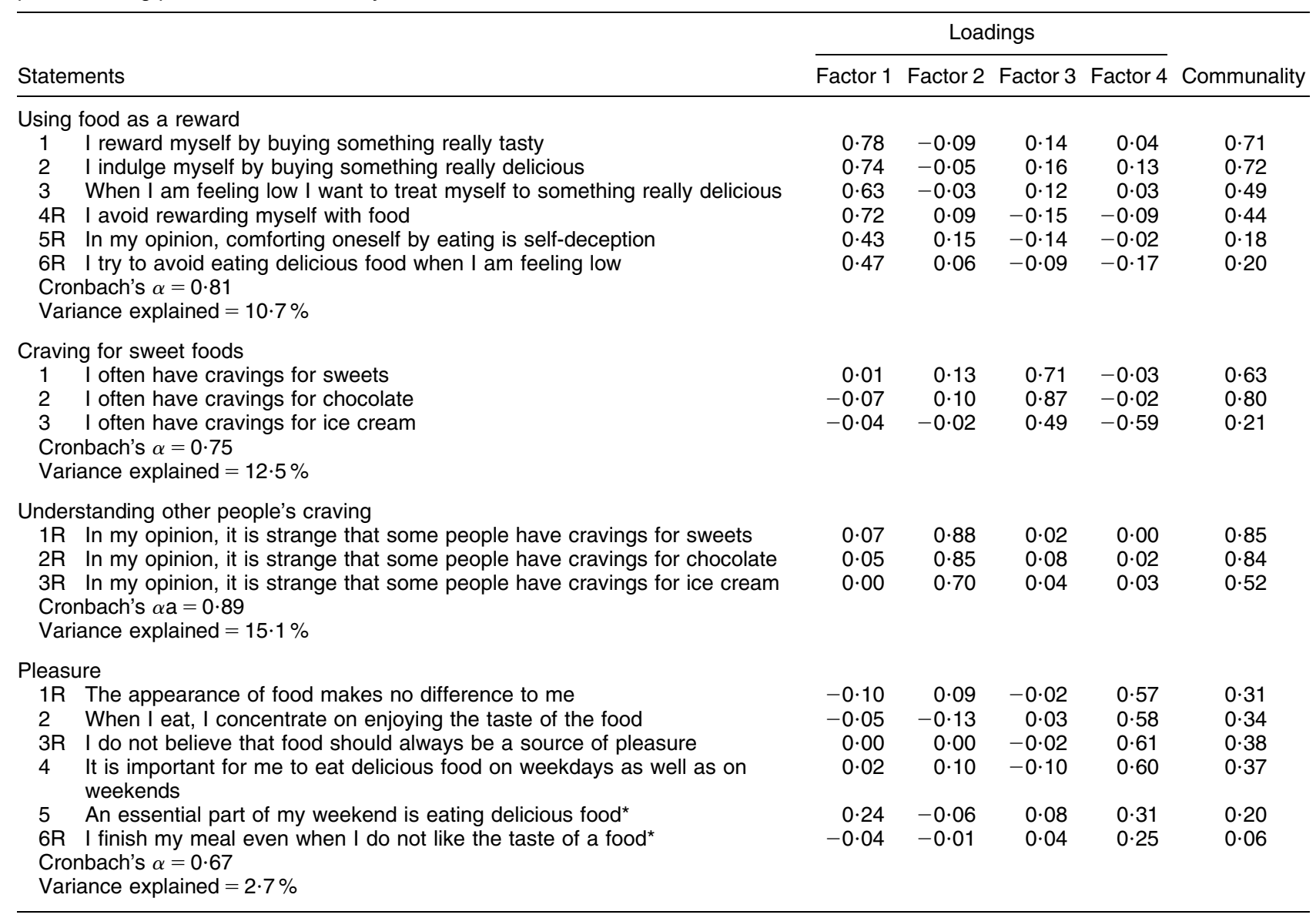

STRIP, Special Turku Coronary Risk Factor Intervention Project for Children.

Reversed statements are marked with an ' $R$ ' after the statement number.

${ }^{*}$ This statement was removed after the first analysis because of the low factor loadings $(<0 \cdot 40)$. The loadings and the communality shown here are from the first analysis.

\section{Associations of eating attitudes with BMI}

'The personal craving for sweet foods' was the only subscale that associated with BMI as $\mathrm{kg} / \mathrm{m}^{2}$. There was a general trend among mothers who had higher BMI to crave more for sweet foods $(P=0.007$ for gender $\times$ BMI interaction, $P=0 \cdot 013$ for mothers' BMI). This association was also seen in the weight group-based analysis in which obese mothers scored higher than normal-weight mothers (difference $=0 \cdot 78, \quad P=0 \cdot 022$ ). Although no other subscale had a linear association with BMI, in the classified analyses elevated scores were detected in 'General health interest' in overweight parents compared with normal-weight parents and obese parents (difference $=1 \cdot 45(P=0 \cdot 04)$ and $2 \cdot 05(P=0 \cdot 01)$, respectively $)$. Further, in 'Light product interest' overweight intervention mothers differed from both normal-weight and obese intervention mothers, as well as from overweight control mothers $(P=0.003$ for gender $\times$ intervention $\times$ weight group interaction; differences were between $2 \cdot 7$ and $3 \cdot 2$ $(P<0 \cdot 005)$ in all analyses for mothers $)$.

\section{Associations between general bealth interest and dietary bebaviour}

Associations between 'General Health interest', nutritional intervention and dietary indicators of healthy eating are presented in Table 4. A high level of 'General health interest' was associated with lower energy-adjusted saturated fat intake, higher energy-adjusted fibre intake and higher fruit and vegetable consumption. Among the intervention parents, the high level of 'General health interest' was also associated with lower energy-adjusted total fat intake. Furthermore, intervention parents had a lower saturated fat intake and a higher $\mathrm{U}: \mathrm{S}$ compared with control parents regardless of the level of 'General health interest'.

\section{Discussion}

In the present study the intervention parents who had taken part in the nutritional intervention aiming to reduce the cardiovascular risk factors of their child were more 
interested in healthy eating compared with the control parents; however, no differences with regard to interest in natural products or hedonic eating attitudes were observed between the intervention and control parents. At the beginning of the study families were randomly allocated to the intervention and control groups, and parents' general eating attitudes, dietary variables indicating healthy eating and BMI did not differ between the groups ${ }^{(9,25)}$. Furthermore, there were no differences in socio-economic status between the intervention and control families ${ }^{(8)}$. Therefore, the results of our study indicate that the intervention effect on dietary behaviour found in the present and previous studies $^{(7-11)}$ may be achieved by enhancing the positive attitude towards healthy eating among the intervention parents. The control parents have also received general dietary information about their child's diet during the study years and they are aware of the fact that they are participating in a heart disease prevention trial. It may thus be speculated that this might influence the control parents' answers and diminish the difference noticed between the intervention and control parents' health attitudes. On the contrary, the 'General health interest' of the control parents was on the same level as that of earlier studies examining the Finnish adult population's attitudes using the HTAS questionnaire (mean values $4 \cdot 5-4 \cdot 7)^{(19,21)}$.

The STRIP parents' 'General health interest' was associated with dietary indicators of healthy eating (i.e. low intake of saturated fat, high intake of fibre and high consumption of fruit and vegetables). This is in accordance with recent Irish and Danish studies ${ }^{(26,27)}$ that showed a link between attitudes towards healthy eating and dietary behaviour as measured by a food record. Individuals who reported conscious efforts to eat a healthy diet or had frequent intentions to eat healthily had a better dietary profile and were more likely to comply with dietary guidelines compared with those with negative attitudes. In our study, higher U:S, an overall indicator of the dietary fat quality, was associated only with the intervention, not with the interest in healthy eating. Further, saturated fat intake was lower among intervention parents than among control parents at every level of interest in healthy eating. This indicates that with a regular but fairly low-impact intervention we managed to influence the main target; that is, the quality of dietary fat, among parents with less interest in healthy eating as well. Although the main focus of the intervention was on the child's diet, parents were encouraged to purchase foods with better fat quality for the whole family and act as role models for children. It appears that small, simple and specific changes in the food choice behaviour of the family, such as buying soft margarine and skimmed milk as suggested repeatedly by the nutritionist in the STRIP intervention, are possible without special interest in healthy eating. There was no intervention effect on fibre intake or fruit and vegetable consumption in our sample. However, the intervention parents had a higher level of 
with backward selection

\begin{tabular}{|c|c|c|c|c|c|c|c|c|c|}
\hline \multirow[b]{3}{*}{ Dietary indicator of healthy eating $\dagger$} & \multicolumn{6}{|c|}{$\mathrm{GHI}^{*}$} & \multirow{3}{*}{$\begin{array}{c}P \text { for interaction } \\
(\mathrm{GHI} \times \text { intervention }) \\
\end{array}$} & \multirow{3}{*}{$\begin{array}{l}P \text { for } \\
\mathrm{GHI}\end{array}$} & \multirow{3}{*}{$\begin{array}{c}P \text { for } \\
\text { intervention }\end{array}$} \\
\hline & \multicolumn{2}{|c|}{$\begin{array}{l}\text { Low } \\
\text { ( } n \text { 173, seventy-eight } \\
\text { intervention parents) }\end{array}$} & \multicolumn{2}{|c|}{$\begin{array}{c}\text { Medium } \\
\text { ( } n \text { 148, ninety-nine } \\
\text { intervention parents) }\end{array}$} & \multicolumn{2}{|c|}{$\begin{array}{c}\text { High } \\
\text { (n 170, } 125 \text { intervention } \\
\text { parents) }\end{array}$} & & & \\
\hline & Mean & SD & Mean & SD & Mean & SD & & & \\
\hline \multicolumn{10}{|l|}{ Total fat intake (\%E) } \\
\hline All & $36 \cdot 6$ & $8 \cdot 8$ & $34 \cdot 0$ & $8 \cdot 3$ & $32 \cdot 8$ & $8 \cdot 3$ & \multirow[t]{3}{*}{$0.034 \S$} & \multirow[t]{3}{*}{$<0.001 \S$} & \multirow[t]{3}{*}{$0.08 \S$} \\
\hline Intervention parents & $38 \cdot 3$ & $9 \cdot 4$ & $34 \cdot 4$ & $8 \cdot 6$ & $31 \cdot 7$ & $8 \cdot 1$ & & & \\
\hline Control parents & $35 \cdot 9$ & $8 \cdot 4$ & $33 \cdot 6$ & $8 \cdot 1$ & $34 \cdot 3$ & $8 \cdot 5$ & & & \\
\hline \multicolumn{10}{|l|}{ Saturated fat intake (\%E) } \\
\hline All & $14 \cdot 6$ & $4 \cdot 3$ & $13 \cdot 3$ & $4 \cdot 5$ & $12 \cdot 4$ & $4 \cdot 3$ & \multirow[t]{3}{*}{ NS } & \multirow[t]{3}{*}{0.003} & \multirow{3}{*}{$<0.001$} \\
\hline Intervention parents & $14 \cdot 3$ & $4 \cdot 3$ & $12 \cdot 6$ & $4 \cdot 3$ & $11 \cdot 5$ & $4 \cdot 0$ & & & \\
\hline Control parents & $14 \cdot 7$ & $4 \cdot 3$ & $14 \cdot 0$ & $4 \cdot 6$ & $13 \cdot 9$ & $4 \cdot 4$ & & & \\
\hline \multicolumn{10}{|l|}{ U:S } \\
\hline All & $1 \cdot 42$ & $4 \cdot 6$ & 1.50 & 0.62 & 1.56 & 0.65 & \multirow[t]{3}{*}{ NS } & NS & \multirow{3}{*}{$<0.001$} \\
\hline Intervention parents & $1 \cdot 56$ & $4 \cdot 6$ & $1 \cdot 67$ & 0.66 & $1 \cdot 69$ & 0.69 & & & \\
\hline Control parents & $1 \cdot 36$ & $4 \cdot 5$ & $1 \cdot 33$ & 0.53 & $1 \cdot 38$ & 0.54 & & & \\
\hline \multicolumn{10}{|l|}{ Fruit and vegetable consumption $(\mathrm{g}) \ddagger$} \\
\hline All & 233.9 & $183 \cdot 0$ & $269 \cdot 4$ & $182 \cdot 0$ & $330 \cdot 7$ & $212 \cdot 0$ & \multirow{3}{*}{ NS } & $<0.001$ & \multirow{3}{*}{ NS } \\
\hline Intervention parents & $253 \cdot 5$ & $205 \cdot 6$ & $276 \cdot 3$ & $198 \cdot 0$ & $328 \cdot 2$ & $212 \cdot 4$ & & & \\
\hline Control parents & $225 \cdot 8$ & $173 \cdot 0$ & $262 \cdot 3$ & $165 \cdot 0$ & $334 \cdot 4$ & $213 \cdot 1$ & & & \\
\hline \multicolumn{10}{|l|}{ Dietary fibre intake (g/MJ) } \\
\hline All & $2 \cdot 25$ & $0 \cdot 88$ & $2 \cdot 51$ & $0 \cdot 87$ & $2 \cdot 72$ & $1 \cdot 05$ & \multirow[t]{3}{*}{ NS } & $<0.001$ & \multirow[t]{3}{*}{ NS } \\
\hline Intervention parents & $2 \cdot 25$ & 0.68 & 2.56 & 0.88 & $2 \cdot 73$ & 1.01 & & & \\
\hline Control parents & $2 \cdot 25$ & 0.95 & $2 \cdot 46$ & $0 \cdot 87$ & $2 \cdot 72$ & $1 \cdot 11$ & & & \\
\hline
\end{tabular}

GHI, General health interest; STRIP, Special Turku Coronary Risk Factor Intervention Project for Children; \%E, percentage of energy; U:S, unsaturated-to-saturated fat ratio.

*GHI was measured using a subscale of the Health and Taste Attitude Scales questionnaire.

Dietary indicators of healthy ealing were measured using a $1 \mathrm{~d}$ food record.

列

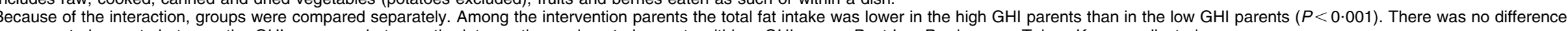
列 
'General health interest' compared with control parents, and higher level of 'General health interest' was associated with higher fibre intake and fruit and vegetable intakes.

The 'Personal craving for sweet foods' was associated with the BMI of mothers who participated in the STRIP study. It is possible that intensive craving for comfortgiving, high-energy-dense foods such as chocolate, sweets and ice cream partly explains the development of overweight, especially in women. Overweight women's tendency to crave for sweet foods may also be a result of dieting and restrained eating, especially in unsuccessful dieters $^{(28)}$. In a cross-sectional study setting it is naturally impossible to draw conclusions about causes and effects. Like in the recent Irish study ${ }^{(2)}$, the STRIP parents' interest in having a healthy diet did not associate linearly with their BMI. However, the overweight parents seemed to be more interested in healthy eating compared with the normal weight or obese parents, and the overweight intervention mothers were more interested in light products compared with the normal-weight or obese intervention mothers.

Parents' 'General health interest' correlated positively with seeking 'Pleasure' in eating, which indicates that people who are interested in eating healthy food are also more interested in the sensory and pleasure-giving qualities of food. It is essential that all the interventions aiming at promotion of healthy eating behaviour also give prime importance to taste preferences and to the pleasure of eating ${ }^{(17)}$. A gender difference in dietary attitudes is well documented $^{(14,19-21,26,29-31)}$. Furthermore, in the present study, mothers scored higher than fathers in both healthrelated and hedonic subscales. Interestingly, attitudes of the intervention fathers towards healthy eating were similar to those of the control mothers; the dietary intervention seemed to compensate the influence of gender in dietary attitudes. In general, women's interest in health and at the same time a positive attitude towards the hedonic aspects of eating may result in women facing more ambivalent and difficult food choice situations compared with men.

In general, the HTAS questionnaire seemed to be a fairly good tool for measuring eating attitudes in adults. The values of Cronbach's $\alpha$ indicated a moderate-to-high reliability of the questionnaire and they corresponded to those obtained earlier when using HTAS in the Finnish population $^{(19,21)}$. However, the number of attitude items in the 'Natural product interest' and 'Pleasure' subscales was reduced in the present study. These subscales also had the lowest level of reliability. Furthermore, the original subscale 'Craving for sweet foods' was divided into two new subscales. Because examination of scree plots is open to interpretation, the four-factor solution for healthrelated items and a three-factor solution for hedonic factors were also examined. The fourth health factor had an eigenvalue close to 1 and it also overlapped with the other factors. Furthermore, the model was not easily interpretable. In the three-factor model for hedonic items, more attitude items needed to be removed compared with the four-factor model.

Our results must be interpreted within the limitations of the chosen methodology. Self-reported explicit measures of attitudes have been criticized for being highly susceptible to social desirability, which often reduces their validity. Instead, the use of implicit measures such as an effective priming paradigm has been suggested for measuring food attitudes $^{(32-34)}$. The respondents of the present study had regularly attended the STRIP study sessions for more than 10 years. We believe that this reduced the respondents' tendency to give socially desirable answers to a certain extent. A further limitation of the present study is the fact that the dietary data were based on a $1 \mathrm{~d}$ food record. However, the participating parents have a long history of filling food records during the STRIP study, which improved the accuracy of recording. Further, we used dietary data to indicate eating behaviour only at a group level, not at an individual level.

In conclusion, the present study indicates that taking part in a regular but fairly low-impact nutritional intervention mainly focused on children's diet is associated with a higher level of interest in healthy eating after 10 years of the intervention. Further, the key target of the dietary intervention (in the STRIP study, the quality of dietary fat) can also be influenced among parents with the lowest level of interest in healthy eating.

\section{Acknowledgements}

The present study was supported by the Juho Vainio Foundation, The Finnish Cardiac Research Foundation, The Yrjö Jahnsson Foundation, The Emil Aaltonen Foundation, the Finnish Cultural Foundation's Regional Funds of Varsinais-Suomi and the Fund from Turku University. The authors have no conflict of interest to declare. S.T. is responsible for the concept and design of the study and for coordinating and accomplishing the data collection, completing the data analysis, designing the statistical analysis and drafting the manuscript; H.L. and L.R. are responsible for the concept and design of the study; L.S. and M.S. designed and performed the statistical analysis; O.S. is responsible for the original STRIP study design. All authors contributed to interpretation of the results and revision of the manuscript. The authors are grateful to all the families who took part in the STRIP study.

\section{References}

1. Gidding SS, Dennison BA, Birch LL et al. (2005) Dietary recommendations for children and adolescents. A guide for practitioners. Consensus statement from the American Heart Association. Circulation 112, 2061-2075.

2. World Health Organization (2003) Diet, Nutrition and the Prevention of Chronic Diseases. Joint WHO/FAO Expert 
Consultation. WHO Technical Report Series no. 916. Geneva: WHO.

3. Mikkilä V, Räsänen L, Raitakari OT et al. (2005) Consistent dietary patterns identified from childhood to adulthood: the Cardiovascular Risk in Young Finns Study. Br J Nutr 93 , 923-931.

4. Mikkilä V, Räsänen L, Raitakari OT et al. (2004) Longitudinal changes in diet from childhood into adulthood with respect to risk of cardiovascular diseases: the Cardiovascular Risk in Young Finns Study. Eur J Clin Nutr 58, 1038-1045.

5. Hill AJ (2002) Developmental issues in attitudes to food and diet. Proc Nutr Soc 61, 259-266.

6. Scaglioni S, Salvioni M \& Galimberti C (2008) Influence of parental attitudes in the development of children eating behavior. Br J Nutr 99, Suppl. 1, S22-S25.

7. Lagström H, Jokinen E, Seppänen R et al. (1997) Nutrient intakes by young children in a prospective randomized trial of a low-saturated fat, low-cholesterol diet. The STRIP baby project. Arch Pediatr Adolesc Med 151, 181-188.

8. Talvia S, Lagström H, Räsänen M et al. (2004) Calorie (energy) and nutrient intakes up to the age of 10 years in the Special Turku Coronary Risk Factor Intervention Project. Arch Pediatr Adolesc Med 158, 41-47.

9. Lagström H, Seppänen R, Jokinen E et al. (1999) Nutrient intakes and cholesterol values of the parents in a prospective randomized child-targeted coronary heart disease risk factor intervention trial. The STRIP baby project. Eur J Clin Nutr 53, 654-661.

10. Niinikoski H, Lagström H, Jokinen E et al. (2007) Impact of repeated dietary counseling between infancy and 14 years of age on dietary intakes and serum lipids and lipoproteins: the STRIP study. Circulation 166, 1032-1040.

11. Talvia S, Räsänen L, Lagström H et al. (2006) Longitudinal trends in consumption of vegetables and fruit in Finnish children in an atherosclerosis prevention study (STRIP). Eur J Clin Nutr 60, 172-180.

12. Rask-Nissilä L, Jokinen E, Rönnemaa T et al. (2000) Prospective, randomized, infancy-onset trial of the effects of a low-saturated-fat, low-cholesterol diet on serum lipids and lipoproteins before school age: the Special Turku Coronary Risk Factor Intervention Project (STRIP). Circulation 102, 1477-1483.

13. Raitakari OT, Rönnemaa T, Järvisalo MJ et al. (2005) Endothelial function in healthy 11-year-old children after dietary intervention with onset in infancy: the Special Turku Coronary Risk Factor Intervention Project for Children (STRIP). Circulation 112, 3786-3794.

14. Räsänen M, Niinikoski H, Keskinen S et al. (2003) Parental nutrition knowledge and nutrient intake in an atherosclerosis prevention project: the impact of child-targeted nutrition counseling. Appetite 41, 69-77.

15. Lennernäs M, Fjellström C, Becker W et al. (1997) Influences on food choice perceived to be important by nationally-representative samples of adults in the European Union. Eur J Clin Nutr 51, Suppl. 2, S8-S15.

16. Steptoe A, Pollard TM \& Wardle J (1995) Development of a measure of the motives underlying the selection of food: the food choice questionnaire. Appetite 25, 267-284.

17. Eertmans A, Baeyens F \& Vanden Berg O (2001) Food likes and their relative importance in human eating behavior: review and preliminary suggestions for health promotion. Health Educ Res 16, 443-456.

18. Kearney JM \& McElhone S (1999) Perceived barriers in trying to eat healthier: results of a pan-EU consumer attitudinal survey. Br J Nutr 81, Suppl. 2, S133-S137.

19. Roininen K, Lähteenmäki L \& Tuorila H (1999) Quantification of consumer attitudes to health and hedonic characteristics of foods. Appetite 33, 71-88.

20. Roininen K \& Tuorila H (1999) Health and taste attitudes in the prediction of use frequency and choice between less healthy and more healthy snacks. Food Qual Prefer 10, 357-365.

21. Roininen K, Tuorila H, Zandstra EH et al. (2001) Differences in health and taste attitudes and reported behaviour among Finnish, Dutsch and British consumers: a cross-national validation of the Health and Taste Attitude Scales (HTAS). Appetite 37, 33-45.

22. Lapinleimu H, Viikari J, Jokinen E et al. (1995) Prospective randomized trial in 1062 infants of diet low in saturated fat and cholesterol. Lancet 345, 471-476.

23. Hakanen M, Lagström H, Kaitosaari T et al. (2006) Development of overweight in an atherosclerosis prevention trial starting in early childhood. The STRIP study. Int J Obes 30, 618-626.

24. World Health Organization (1998) Obesity: Preventing and Managing the Global Epidemic. Geneva: WHO.

25. Saarilehto S, Keskinen S, Lapinleimu H et al. (2001) Connections between parental eating attitudes and children's meagre eating: questionnaire findings. Acta Paediatr 90, 333-338.

26. Hearty AP, McCarthy SN, Kearney JM et al. (2007) Relationship between attitudes towards healthy eating and dietary behaviour, lifestyle and demographic factors in a representative sample of Irish adults. Appetite 48, 1-11.

27. Biltoft-Jensen A, Groth MV, Matthiessen J et al. (2008) Diet quality: associations with health messages included in the Danish Dietary Guidelines 2005, personal attitudes and social factors. Public Health Nutr 12, 1165-1173.

28. Moreno S, Warren CS, Rodríguez S et al. (2009) Food cravings discriminate between anorexia and bulimia nervosa. Implications for 'success' versus 'failure' in dietary restriction. Appetite 52, 588-594.

29. Barker ME, Thompson KA \& McClean SI (1995) Attitudinal dimensions of food choice and nutrient intake. Br J Nutr 74, 649-659.

30. Kearney M, Kearney JM, Dunne A et al. (2000) Sociodemographic determinants of perceived influences on food choice in a nationally representative sample of Irish adults. Public Health Nutr 3, 219-226.

31. Nestle M, Wing R, Birch L et al. (1998) Behavioral and social influences on food choice. Nutr Rev 56, 5 Pt 2, S50-S74.

32. Czyzewska M \& Reiko Graham R (2008) Implicit and explicit attitudes to high- and low-calorie food in females with different BMI status. Eat Behav 9, 303-312.

33. Lamote S, Hermans D, Baeyens F et al. (2004) An exploration of affective priming as an indirect measure of food attitudes. Appetite 42, 279-286.

34. Verhulst F, Hermans D, Baeyens F et al. (2006) Determinants and predictive validity of direct and indirect measures of recently acquired food attitudes. Appetite $\mathbf{4 6}$, $137-143$. 